\title{
El derecho administrativo global $y$ las fuentes del derecho administrativo
}

\author{
Global administrative law and the sources \\ of administrative law
}

Direito administrativo global e s fontes do Direito administrativo

Le droit administratif global et les sources du droit administratif 全球行政法和行政法洲源

Benjamín Marcheco Acuña ${ }^{1}$ Universidad de La Habana, Raudel Navarro Hernández ${ }^{2}$ Cuba

Revista Derechos en Acción ISSN 2525-1678/ e-ISSN 2525-1686

Año 4/Nº 13, Primavera 2019 (21 septiembre a 20 diciembre), 154-189

DOI: https://doi.org/10.24215/25251678e335

ORCID: https://orcid.org/0000-0002-1967-9530

https://orcid.org/0000-0001-9107-7887

Recibido: 07/07/2019

Aprobado: 15/09/2019

Resumen: La regulación administrativa global, más allá de la derivada de los tratados internacionales, constituye hoy sin dudas una manifestación reciente del Derecho Administrativo en la medida en que este, como cualquier otra rama del derecho, se ha visto afectado por el fenómeno de la globalización. De la configuración teórica del Derecho Administrativo Global y en particular sobre sus relaciones con el derecho interno se ha escrito poco en Latinoamérica, pese a su manifestación práctica y común en las regulaciones jurídicas de los

\footnotetext{
1 Doctor en Ciencias Jurídicas (Universidad de La Habana). Profesor de Derecho. Universidad de Guayaquil: benjamacheco@gmail.com

2 Master en Derecho Constitucional y Administrativo, Universidad de La Habana, Abogado. raudelnhdez1986@gmail.com
} 
diferentes sectores en que se filtra el Derecho Administrativo. Con el presente trabajo se pretenden sistematizar las principales corrientes, concepciones y teorías sobre el desarrollo de las funciones administrativas en el contexto internacional, determinando particularmente las formas y modalidades de la actividad regulatoria global, como un primer acercamiento a esta reciente expresión del Derecho Administrativo.

Palabras claves: Derecho Administrativo Internacional, Derecho Administrativo Global, administración global, espacio administrativo global, fuentes del Derecho Administrativo.

Abstract: The global administrative regulation, beyond that derived from international treaties, is a recent manifestation of Administrative Law insofar as this, like any other branch of law, has been affected by the phenomenon of globalization. About the theoretical configuration of global administrative law and in particular its relations with domestic law has been written very little in Latin America, despite its practical and common in the legal regulations of the different sectors involved administrative law manifestation. With this paper we intend to systematize the mainstream, concepts and theories on the development of administrative functions in the international context, particularly determining the forms and modalities of global regulatory activity, as a first approach to this recent expression of Administrative Law.

Keywords: International administrative law, global administrative law, global administration, global administrative space and sources of administrative law.

Resumo: A regulamentação administrativa global, além da derivada dos tratados internacionais, hoje constitui sem dúvida, uma manifestação recente do Direito Administrativo na medida em que este, como qualquer outro ramo do direito, foi afetado pelo fenômeno da globalização. Da configuração teórica do Direito Administrativo Global e em particular de suas relações com o direito interno, pouco foi escrito na América Latina, apesar de sua manifestação prática e comum nos regulamentos legais dos diferentes setores em que escoa-se o Direito Administrativo. Com este trabalho pretendemos sistematizar as principais tendências, concepções e teorias sobre o desenvolvimento de funções administrativas no contexto internacional, determinando 
particularmente as formas e modalidades da atividade reguladora global, como uma primeira abordagem a esta recente expressão do Direito Administrativo.

Palavras-chave: Direito Administrativo Internacional, Direito Administrativo Global, administração global, espaço administrativo global, fontes do Direito Administrativo

Résumé: La régulation administrative globale, au-delà de celle qui vient des traités internationaux, constitue aujourd'hui sans aucun doute une manifestation récente du droit administratif dans la mesure où celui-ci, comme toutes les autres branches du droit, a été affecté par le phénomène de la Globalisation. En ce qui concerne la configuration théorique du droit administratif mondial et en particulier sur ses relations avec le droit interne, peu de choses ont été écrites en Amérique latine, malgré sa manifestation pratique et commune dans les réglementations juridiques des différents secteurs dans lesquels le droit administratif se manifeste. Avec ce travail, nous avons l'intention de systématiser les principales tendances, conceptions et théories sur le développement des fonctions administratives dans le contexte international, en particulier en déterminant les formes et les modalités de l'activité réglementaire mondiale, comme première approche à cette expression récente du droit administratif.

Mot-clés: Droit Administratif International, Droit administratif Global, Administration Globale, espace administratif global, sources du Droit Administratif.

摘要: 毫无疑问, 全球行政规章已超越了国际条约, 今天无疑构成了 行政法的最新体现, 其程度与其他法律分支一样都受到了全球化现 象的影响。在全球行政法的理论结构中, 特别是关于其与国内法的 关系, 尽管拉丁美洲在行政法被过滤的不同部门的法律法规中具有 实践性和共同性表现, 但在拉丁美洲几乎没有写成。通过这项工作, 我们打算将国际背景下行政职能发展的主要趋势, 概念和理论系统 化, 特别是确定全球监管活动的形式和方式, 作为最近表达行政法 的第一种方法。

关键字: 国际行政法, 全球行政法, 全球行政, 全球行政空间, 行政法 渊源。 


\section{Introducción}

Cuando, por lo general, tanto en la enseñanza como en la propia práctica del Derecho se trata el tema de las fuentes (formales) del Derecho Administrativo, es prácticamente nula la referencia a la regulación administrativa global, a excepción, como hemos dicho, de las derivadas de los tratados internacionales como fuente directa del Derecho Administrativo.

Ello es así, fundamentalmente, porque aún se entiende al Derecho Administrativo desde lo que el profesor Javier BARNÉs denomina "los tres diques que delimitaban sus señas de identidad: como derecho nacional, derecho del poder, y como derecho de vocación ejecutiva"3 y porque el Derecho Administrativo Global, es aún una disciplina en ciernes.

Sin embargo, es evidente que, en los últimos años, el fenómeno de la globalización y sus manifestaciones concretas, como las regulaciones de alcance supranacional, han producido importantes innovaciones estructurales y en las formas de actuación de la Administración pública; lo que ha abocado al Derecho Administrativo a un replanteamiento de sus principales postulados teóricos.

En el Derecho Administrativo actual se ha sentido la notable influencia de la regulación administrativa global, producto de un aumento en el alcance y las formas de regulación y administración que se desarrollan más allá de las fronteras estatales para abordar las consecuencias de la interdependencia generada por el fenómeno de la globalización. ${ }^{4}$

A través de sus distintas modalidades regulatorias (tanto las clásicas fuentes del Derecho Internacional Público de naturaleza vinculante como las del denominado soft law en sus distintas formas que derivan su capacidad regulatoria de su alto

\footnotetext{
3 BARnÉs, Javier. Transformaciones (científicas) del Derecho administrativo. Historia y retos del Derecho administrativo contemporáneo, Global Law Press, Sevilla, 2012, p. 10.

4 Marcheco Acuña, Benjamín. "Los desafíos de la ciencia del derecho administrativo ante la globalización", Revista General de Derecho Administrativo, No. 49, 2018, p. 4.
} 
contenido técnico y su fuerza persuasoria) penetran en los sistemas normativos nacionales, ya sea determinando el contendido normativo de las fuentes jurídicas nacionales, ya condicionando la actuación de las autoridades como línea orientadora para la mejor toma de decisiones o como criterio interpretativo del derecho positivo. ${ }^{5}$

Son numerosas las entidades y organismos normativos globales cuyos estándares se incorporan a los ámbitos reguladores nacionales en distintos niveles y respetadas en la práctica pese a no ser formalmente vinculantes precisamente por las presiones del entorno altamente competitivo y por la supervisión constante de su cumplimiento. Por otro lado, los propios actores privados (individuos, grupos, corporaciones, ONGs), destinatarios de esos regímenes regulatorios globales son, de manera creciente, sujetos directos de las acciones ejecutivas o aplicación de medidas con efectos jurídicos directos, sin necesidad, en algunos casos de intervención de autoridades internas, con lo cual se va acortando paulatinamente la distancia que entre unos y otros ha impuesto el Derecho Internacional Público tradicional (p. ej., las decisiones sobre seguridad internacional del Consejo de Seguridad de la ONU, que afectan tanto a países concretos - mediante sanciones-, como sobre las personas -la lista de personas consideradas amenazas a la paz mundial).

El fenómeno descrito afecta indudablemente, a más de las instituciones clásicas del Derecho Administrativo, sus conceptos y técnicas, a su sistema de fuentes; tanto en los procedimientos de producción, en el contenido normativo como, fundamentalmente, en su interpretación y aplicación.

\footnotetext{
5 En este sentido resulta muy ilustrativa la paradigmática sentencia Grimaldi, de 13-12-1989, del Tribunal de Justicia de la Unión Europea, quien entiende que este tipo de regulaciones, a pesar de carecer de eficacia vinculante, "no pueden ser considerados como carentes en absoluto de efectos jurídicos" y los jueces están obligados a tenerlas en cuenta a la hora de resolver los litigios de que conocen, "sobre todo cuando aquéllas ilustran acerca de la interpretación de disposiciones nacionales adoptadas con el fin de darles aplicación, o también cuando tienen por objeto completar las disposiciones comunitarias dotadas de fuerza vinculante".
} 
Con el presente trabajo se pretenden sistematizar las principales corrientes, concepciones y teorías sobre el desarrollo de las funciones administrativas en el contexto global, determinando particularmente las formas y modalidades de la actividad regulatoria global y su incidencia en el sistema de fuentes del Derecho Administrativo.

\section{I.1. El Derecho Administrativo Global: denominaciones, sentido y alcance}

En el lenguaje jurídico tradicional, el adjetivo "internacional" ha estado siempre asociado a aquéllas ramas del derecho positivo que se refieren a las relaciones entre los Estados como sujetos del Derecho Internacional Público, o entre los particulares como sujetos del Derecho Internacional Privado; si bien cada una de dichas ramas tiene sus propias particularidades, su nota común es que se refieren a la elaboración, interpretación o aplicación de leyes que exceden los límites de un Estado en particular. ${ }^{6}$

$\mathrm{Al}$ resto de las ramas del derecho las une, por encima de cualquiera de las diferencias relevantes, su cualidad de estar restringidas, tanto en su origen como en su aplicación, a los límites del Estado nacional cuyas leyes e instituciones son expresión de su soberanía. De ahí que la expresión "Derecho Administrativo Internacional" u otras que aparecen a primera vista como sinónimos, no dejan de despertar el interés para los investigadores acostumbrados a concebir el Derecho Administrativo como un conjunto de normas e instituciones básicamente nacionales.

\footnotetext{
6 Además de esa gran distinción entre dos campos fundamentales del Derecho internacional, el sustantivo suele aplicarse a otras ramas como el Derecho comercial internacional, el Derecho económico internacional y el Derecho penal internacional. Sobre los dos primeros puede verse Fernández RozAS, José Carlos. "El Derecho del comercio internacional en el contorno de la globalización." Escriva. Revista del Colegio de Notarios del Estado de México, No. 5/2000, pp. 161-230. Sobre el tercero puede verse BAssIounI, Cherif. "El Derecho penal internacional: Historia, objeto y contenido" Anuario de Derecho Penal y Ciencias Penales, Madrid, 1982, pp. 5- 42, y EstupIÑan Siıva, Rosmerlín. "Principios que rigen la responsabilidad internacional penal por crímenes internacionales." UNAM-Instituto de Investigaciones Jurídicas. Anuario Mexicano de Derecho Internacional, vol. XII, 2012, pp. 133-173.
} 
No obstante, en vista de que la expresión es de uso común ya en ciertos sectores de los estudios jurídicos, conviene precisar cuál es su sentido y alcance, así como las expresiones análogas, los sujetos y el objeto de una posible rama del saber jurídico que aún se considera en construcción o emergente. ${ }^{7}$

Para ello es preciso, en primer lugar, fijar una definición básica del Derecho Administrativo que tome en cuenta sus elementos más importantes, para luego analizar en qué se diferencia éste del denominado Derecho Administrativo Internacional y cuáles son las mutuas influencias que se pueden establecer entre ambos.

En 1950, el jurista cubano Julián Modesto Ruz definió el Derecho Administrativo como "el conjunto de principios y reglas que rigen la acción organizada de los funcionarios y empleados públicos encaminada a la realización directa, inmediata, y casi siempre concreta de determinadas conveniencias de interés común." ${ }^{\prime \prime}$

Varios años más tarde, Héctor Garcini Guerra concretó su concepción del Derecho Administrativo al definirlo como "aquella rama jurídica que fija los principios y analiza las normas que orientan y regulan las relaciones sociales que se producen en la organización y en la actividad de la Administración del Estado considerada en todas sus esferas, tanto nacional como local."

En ambas definiciones se aprecia la coincidencia de que el Derecho Administrativo está integrado por normas y principios

\footnotetext{
7 Que es un sector de estudios "en construcción" es con seguridad la única nota común a los estudiosos que se dedican al tema. Cfr. Fernández Lamela, Pablo. “Globalización y Derecho público. Introducción al Derecho administrativo internacional." En Cienfuegos Salgado, David y López Olvera, Miguel Alejandro (coords.) Estudios en homenaje a Don Jorge Fernández Ruiz. Tomo I Derecho Administrativo, México D.F., UNAM, 2005, p. 45: "a fin de introducirnos a su estudio, identificaremos algunos de sus elementos y tópicos básicos, dejando en claro que cualquier conclusión que se exponga al respecto sólo puede tener carácter provisorio, dado que se trata de un tema en constante evolución y aún no consolidado." pp. 45- 63.

8 Ruzz, Julían M. Principios de Derecho Administrativo. Escuela de Ciencias Comerciales, La Habana, Universidad de la Habana, 1950, p. 14.

9 Garcinı GuerRa, Héctor. Derecho Administrativo. Editorial Pueblo y Educación, La Habana, 1986 , p. 27
} 
que regulan la organización y funcionamiento de la Administración pública estatal, tanto a nivel nacional como local en la definición de H. Garcins. En el caso de los principios, que son resultado de la labor doctrinal antes que, de decisiones legislativas, su alcance puede ir más allá del ámbito nacional, puesto que por su generalidad y abstracción pueden ser aplicados al análisis del Derecho Administrativo en diferentes ordenamientos jurídicos.

Algo muy diferente sucede con las normas jurídicas, que solo son aplicables hasta donde llega la esfera de influencia territorial de la entidad estatal que la emitió, y es aplicable solo dentro de los límites de su competencia. En síntesis, en ninguna de las dos definiciones el Derecho Administrativo tiene un alcance más allá de los límites del Estado.

El análisis anterior se refiere a lo que Catalina Escuín PaloP identifica como el concepto tradicional del Derecho Administrativo, que considera se funda en tres presupuestos básicos: i)- la Administración concebida a partir del principio de la división de poderes, definiéndose el Derecho que regulaba la actividad del Poder Ejecutivo por su carácter residual (ni legislación, ni jurisdicción); ii)- la Administración como una unidad monolítica y autárquica, organizada de acuerdo con un criterio centralista, regida por el principio de jerarquía y; iii)- la actividad administrativa como una actividad formalizada, siempre subsumible en las previsiones de la Ley. ${ }^{10}$

Probablemente la mejor expresión para definir una concepción del Derecho Administrativo basada en aquellos presupuestos es la afirmación de Eduardo García de EnTERría y Tomás-Ramón FernánDEZ, cuando afirman que "es lícito decir que el Derecho Administrativo es el Derecho Público interno por excelencia del Estado. ${ }^{11}$

\footnotetext{
10 Escuin Palop, Catalina. Curso de derecho administrativo, parte general. Tirant lo Blanch, Valencia, 2011, p. 19.

11 García de Enterría, Eduardo y Fernández, Tomás R. Curso de Derecho Administrativo I, $15^{\mathrm{a}}$ ed., Madrid, Civitas-Thomson Reuters, 2011, p. 49.
} 
Ante esa característica de derecho interno por excelencia, cabe preguntarse qué relaciones se pueden establecer entre el Derecho Administrativo y el Derecho Internacional; esas relaciones se analizan usualmente en el tema específico de las fuentes del Derecho Administrativo, donde se incluyen los tratados internacionales cuyas formas de recepción en el derecho interno son definidas a nivel constitucional. ${ }^{12}$

$\mathrm{Al}$ margen de las formas concretas de recepción de tratados y las diferentes posiciones teóricas respecto a su carácter de fuentes del Derecho Administrativo, en las últimas décadas se ha ido avanzando más allá de las relaciones entre el Derecho Internacional y el Derecho Administrativo, lo que ha tenido como unos de sus resultados más publicitados la constitución de un llamado Derecho Administrativo Internacional ${ }^{13}$ o Derecho Administrativo Global, del que se pueden encontrar indicios en algunos estudios de las primeras décadas del siglo $\mathrm{XX}$, sin que ello diera lugar a la configuración de una nueva rama jurídica como se pretende en los estudios actuales. ${ }^{14}$

En términos de principios pereciera que no existe ninguna diferencia sustancial entre las expresiones Derecho Administrativo Internacional y Derecho Administrativo Global; pero una revisión más atenta permite apreciar que ambas expresiones tiene sentidos y alcances distintos: la primera, al menos en los estudios tradicionales del Derecho Administrativo, se refiere a los puntos de convergencia en la aplicación del derecho interno con el derecho internacional en sus aspectos netamente

12 Un análisis del tema en el Derecho español puede verse en GARCía dE ENTERRía, Eduardo y Fernández, Tomás R., op. cit. pp. 159 y siguientes.

13 Cfr. Schmidt-Assmann, Eberhard. "La ciencia del derecho administrativo ante el reto de la internacionalización de las relaciones administrativas." Revista de Administración Pública No. 171/2006, Madrid: "por derecho administrativo internacional se entiende el derecho conflictual jurídico-público, esto es, el Derecho nacional de selección de la ley aplicable a supuestos de hecho dotados de conexión con el extranjero." P. 32. págs. 7-34

14 Un análisis del contexto del surgimiento y desarrollo del Derecho administrativo global puede verse en López Escarcena, Sebastián. “Contextualizando el derecho administrativo global." Anuario Colombiano de Derecho Internacional, Vol. 11/2018, pp. 259-305. 
administrativos; ${ }^{15}$ mientras que el segundo se refiere tanto a las normas como a las instituciones internacionales cuyas disposiciones, aunque no se basen en tratados internacionales, afectan la administración pública interna. ${ }^{16}$

Aparte de esa distinción de principios, en los estudios actuales se usan indistintamente ambas denominaciones para hacer referencia a un conjunto de estudios, análisis y teorías que, si bien no forman aún un cuerpo de doctrina homogéneo, ${ }^{17}$ tiene como objeto de estudio común las prácticas regulatorias y decisorias de instituciones públicas o privadas de carácter internacional y con orígenes diversos.

Metodológicamente, esos estudios "describen el fenómeno de la desaparición gradual de las tradicionales fronteras entre lo internacional y lo nacional, y entre lo público y lo privado; $y$, por otro, critican el sistema de fuentes y sujetos que históricamente han regido al derecho internacional, que incluye

15 En su tiempo, GarCINI GuerRa distinguía entre un “Derecho Internacional Administrativo que regula las relaciones derivadas de la existencia de una Sociedad Internacional y un Derecho Administrativo Internacional que regula la actividad del Estado enderezada a la realización de sus fines más allá de sus fronteras nacionales." Cfr. op. cit. p. 35.

16 Un análisis sobre las diferencias específicas entre el Derecho administrativo internacional y el Derecho administrativo global puede verse en Brewer-CARíAs, Allan R. "El Derecho administrativo global en materia de cooperación policial y el procedimiento administrativo global desarrollado ante interpol, como administración global, para la protección de las personas." Ponencia en el Tercer Congreso Internacional de Derecho Administrativo, sobre "Derecho Administrativo Global" organizado por el Instituto de Investigaciones Jurídicas de la Universidad Nacional Autónoma de México y la Facultad de Derecho y Criminología de la Universidad Autónoma de Nuevo León, Monterrey, Nuevo león, 23-25 abril 2009, p 3 y siguientes. Disponible en http://allanbrewercarias. com/wp-content/uploads/2009/04/1045.-1-990.-PROCEDIMIENTO-ADMINISTRATIVOGLOBAL-EN-MATERIA-DE-POLIC\%C3\%8DA.-Monterrey.doc.pdf. Consultado el 5 de julio de 2018.

17 "Los modelos de gobernanza global que han surgido han sido moldeados por un poco evidente pero importante y creciente cuerpo de normas de derecho administrativo global. Este cuerpo de normas no se encuentra hoy en día unificado- de hecho, no es todavía un campo organizado en la academia o en la práctica." Cfr. Kingsbury, Benedict, et al. "El Surgimiento del Derecho Administrativo Global," Revista de Derecho Público No. 24/2010, Bogotá, Facultad de Derecho Universidad de los Andes, p. 23. 
principalmente a tratados y costumbres, así como a Estados y organizaciones internacionales." ${ }^{18}$

Siendo así, es posible afirmar que los actuales estudios que se refieren al Derecho Administrativo Internacional, no se circunscriben únicamente a la evolución interna del Derecho Administrativo tradicional, sino que parten de las limitaciones de éste para explicar adecuadamente la ampliación de los sujetos que en calidad de reguladores o decisores internacionales, inciden y hasta modifican el orden jurídico interno, sin estar amparados por las tradicionales fuentes de legitimación como son los tratados internacionales.

Esa ampliación de los sujetos del Derecho Administrativo implica, además la necesidad de delimitar un ente al que se pueda identificar como "Administración global", el espacio sobre el que ejerce sus funciones, el llamado "espacio administrativo global"; ambas cosas son imprescindibles en la medida en que "la conceptualización del derecho administrativo global presupone la existencia de una administración global o transnacional." ${ }^{19}$

No obstante la superposición de estudios, conceptos y análisis sobre el Derecho Administrativo Global, una primera sistematización apunta a ciertas diferencias que se pueden apreciar entre los estudios realizados en Estados Unidos y los países europeos; esa diferencia consiste en que mientras que en Estados Unidos la escuela del Derecho Administrativo Global es más pragmática, privilegiando los aspectos procesales del Derecho Administrativo Global, en Italia y Francia, así como en España, es más teórica, estudiando preferentemente lo sustantivo de este derecho. ${ }^{20}$

En cualquier caso, los estudios de Derecho Administrativo Global parten de la constatación empírica de diversos elementos, que pueden resumirse en la internacionalización de la

\footnotetext{
18 Cfr. López Escarcena, Sebastían. "Contextualizando el derecho administrativo..." op. cit. p. 261. Anuario Colombiano de Derecho Internacional, Vol. 11/2018, pp. 259-305.

19 Kingsbury, Benedict. et al. "El Surgimiento del Derecho Administrativo..." op. cit. p. 8.

20 Cfr. López Escarcena, Sebasían. “Contextualizando el derecho administrativo... " op. cit. p. 271.
} 
Administración, definida a grandes rasgos como "todos aquellos fenómenos de carácter administrativo que sobrepasan las fronteras de los espacios administrativos nacionales, tanto si proceden de los mismos como si han sido concebidos desde un primer momento sin consideración de tales fronteras". ${ }^{21}$

En síntesis, se trata de la existencia de instituciones, regulaciones y procedimientos de carácter internacional o global que influyen en la Administración pública nacional, sin que necesariamente haya un tratado internacional que las legitime y haga vinculantes, creando así un espacio de relaciones internacionales de gran complejidad, en las que intervienen sujetos reguladores tradicionales junto a otros emergentes.

En una visión de conjunto y como conclusión, puede decirse que "el ámbito regulatorio global se sitúa en medio del derecho internacional y el derecho administrativo, superando la dicotomía o dualidad conceptual nacional-internacional o interior-exterior, para constituir así espacios y tipos de interacciones diferentes que necesitan del desarrollo de ciertos conceptos y principios que den forma y legitimidad a esta nueva regulación." 22

Ese lugar intermedio entre el Derecho Administrativo y el Derecho Internacional, punto de convergencia donde se ubica el Derecho Administrativo Global, permite fijar sus características principales; se trata de un Derecho Global porque sus normas rigen sin consideración de fronteras; sus efectos rebasan o desbordan los rígidos confines del Estado; porque se ocupa del ejercicio del poder por una serie de organizaciones que no pertenecen a ningún Estado en particular; le reconoce a los particulares la condición de sujetos de derecho en el plano internacional; sus normas limitan o condicionan la actividad

\footnotetext{
21 Cfr. Schmidt-Assmann, Eberhard. "La ciencia del derecho... " op. cit. p. 9.

22 Cfr. Gambardella d’Etigny, Maité y von Loebenstein Weil, Beatriz. Recensión al libro Hacia el Derecho Administrativo Global: fundamentos, principios y ámbito de aplicación de Benedict KingsbuRy y Richard B. Stewart (Global Law Press, Sevilla, 2016). En Revista de Derecho Público, Universidad de Chile, vol. 84/2016, pp. 179- 183.
} 
de la Administración nacional; porque sus normas, en síntesis, autorizan a los particulares cuyos derechos resultaren afectados por la actuación de la Administración para demandar en ultramar el control jurisdiccional de los actos que les causan gravamen. ${ }^{23}$

Finalmente, Allan R. BREwER-Carías expone una definición de Derecho Administrativo que incluye tanto al global como el nacional: "el Derecho Administrativo es el instrumento normativo destinado a garantizar el equilibrio entre los poderes atribuidos a la Administración Global, las atribuciones de las Administraciones nacionales y los derechos de los particulares; en definitiva, garantizar la sujeción del Estado al derecho, al punto de que sin Estado de Derecho (rule of law), no habría derecho administrativo;" en ese contexto el Derecho Administrativo Global es "un derecho que regula a Administraciones globales que ya no son parte del Estado o de un Estado nacional."24

Sin embargo, pese a la creciente utilización del término Derecho Administrativo Global y los esfuerzos por constituirlo en una nueva rama del derecho, una nueva ciencia jurídica o un ordenamiento jurídico singular, María del Pilar CHÁvez escribe sobre la "inexistencia del derecho administrativo global" y considera que "la utilización del concepto es errante. En ocasiones se recurre a él para demonizar al derecho administrativo de las organizaciones internacionales, mientras que en otras viene a resumir el derecho aplicado por determinados tribunales arbitrales, quedando por ello su contenido a merced del doctrinario que lo enuncia." 25

23 Cfr. Muci BorJas, J. A. "Control Judicial y Arbitraje Internacional conforme al Derecho Administrativo Global", Caracas, Centro de Arbitraje de la Cámara de Comercio de Caracas, 2005, p. 11.

24 Brewer-Carías, Allan. R. "El Derecho administrativo global..." op. cit. p. 2.

25 Chávez, María P. "La inexistencia del derecho administrativo global." Diario Administrativo No. 76 de 4 de agosto de 2015, señala que "la denominación encierra una contradicción en sí misma, toda vez que el derecho administrativo es por definición inherente al Estado moderno y el adjetivo "global" parece ampliar su ámbito de aplicación espacial por fuera de 


\section{I.2. Los elementos del Derecho Administrativo Global: sujetos reguladores; sujetos de regulación, estructura y funcionamiento}

Al ser el Derecho Administrativo global un tipo de derecho en formación y en constante expansión, ${ }^{26}$ una de las principales tareas que deben realizar sus estudiosos es la determinación de los elementos principales que lo configuran, aquellos en torno a los cuales giran tanto los sujetos reguladores y objetos de regulación, como las propias normas y procedimientos de acuerdo con los que funcionan.

A. Sujetos reguladores. Para comprender quiénes o cuáles son los sujetos reguladores del Derecho Administrativo Global, conviene sistematizar las principales formas de internacionalización de las relaciones administrativas; de acuerdo con el análisis realizado por SchmidT-Assmann esas formas pueden ser reducidas a cuatro.

i) formas de cooperación bilateral;

ii) formas organizativas regionales;

iii) formas organizativas de alcance mundial;

iv) redes informales de autoridades.

Por su parte, Kingsbury, Krisch y Stewart distinguen "cinco tipos principales de regulación administrativa globalizada:" ${ }^{27}$

i) administración por parte de organizaciones internacionales formales;

las fronteras de aquel hasta los confines de la tierra. En el estado actual del derecho internacional no existe cohesión normativa más allá de las normas denominadas de iuscogens y de parte del derecho derivado de las directivas del Consejo de Seguridad tomadas en el marco del capítulo VII de la Carta de las Naciones Unidas. En consecuencia, no es posible hablar -en términos sistémicos- de un derecho administrativo que tenga escala global."

26 Gordillo, Agustín. Tratado de Derecho Administrativo y obras selectas, Buenos Aires, Fundación de Derecho Administrativo, 2017, t-I, p. V-14.

27 Cfr. Kingsbury, B. et al. "El Surgimiento del Derecho Administrativo Global..." op. cit. p. 9. Según los autores, "en la práctica, muchas de estas capas se superponen o se combinan, pero nosotros proponemos esta selección de tipos ideales para facilitar investigaciones futuras." 
ii) administración basada en la acción colectiva de redes transnacionales de acuerdos de cooperación entre funcionarios reguladores nacionales;

iii) administración distribuida llevada a cabo por reguladores nacionales bajo tratados, redes $\mathrm{u}$ otros regímenes cooperativos;

iv) administración por acuerdos híbridos intergubernamentales privados;

v) administración por instituciones privadas con funciones regulatorias.

Si alguna diferencia tiene el Derecho Administrativo Global con respecto a las formas tradicionales en que se han resuelto las convergencias entre el Derecho Administrativo y el Derecho Internacional, no radica precisamente en la existencia de todas esas formas diversas de administración llamadas global, puesto que entre las que señalan los autores solo las identificadas como redes informales de autoridades, o la administración por instituciones privadas con funciones regulatorias, quedan al margen de los tratados internacionales.

Algo muy distinto sucede cuando, por ejemplo, las instituciones u organizaciones internacionales de carácter político asumen funciones de reguladores internacionales en materia administrativa, o adoptan regulaciones que deben ser aplicadas por parte de los Estados en su derecho interno, o son las propias instituciones internacionales quienes las ejecutan en el territorio de algún Estado.

Dicho de otra manera, la existencia de un Derecho Administrativo Global radica, por un lado, en la ampliación de los sujetos de regulación administrativa internacional y, por otro, en la existencia de sujetos reguladores no derivados necesariamente de los tratados o convenios internacionales y que, sin embargo, disponen de poderes de hecho para conseguir que sus regulaciones sean incorporadas o aplicadas en el Derecho Administrativo interno. 
Los dos aspectos que sirven de fundamento al Derecho Administrativo Global deben ser analizados en un contexto particularmente complejo, que se caracteriza según CASSESE por la globalización, la privatización, la participación ciudadana, o las nuevas responsabilidades fiscales a nivel global, el crecimiento de los poderes ultra-nacionales e intra-nacionales, el aumento de la separación funcional, en la que los Gobiernos nacionales actúan tanto con poderes soberanos como con poderes delegados de organismos ultra-nacionales, el aumento de la comunicación entre los ordenamientos jurídicos nacionales, el crecimiento de principios compartidos por varios ordenamientos jurídicos, a nivel mundial, nacional y local, entre otros. ${ }^{28}$

¿Cuáles son esos sujetos reguladores propios del Derecho Administrativo Global, que funcionan en ese contexto y con base en los presupuestos señalados? Más allá de las referencias a instituciones concretas, a organismos, instituciones públicas o privadas o redes internacionales, los estudios sobre la materia no disponen aún de una descripción analítica que permita incluir o excluir instituciones u organismos, con independencia de los ejemplos concretos.

Según Kingsbury et. al. "los órganos globales administrativos incluyen órganos regulatorios intergubernamentales formales, redes regulatorias informales intergubernamentales y arreglos de coordinación, órganos regulatorios nacionales que operan en relación con un régimen internacional intergubernamental, órganos regulatorios híbridos público-privados, y algunos órganos regulatorios privados que ejercen funciones de gobernanza trasnacional de importancia pública específica." ${ }^{29}$

\footnotetext{
28 A raíz de esos fenómenos han "surgido nuevos conceptos e ideas que han penetrado en la literatura: Ia Nueva Gestión Pública, la gobernanza, la rendición de cuentas [accountability], los organismos expertos, la dirección [steering]. Pero, como la continuidad y el cambio van de la mano, es difícil estudiar este mosaico de contradicciones utilizando viejos métodos." Cfr. Cassesse, Sabino. "New Paths for Administrative Law... "op. cit. pp. 3- 4.

29 Kingsbury, B. et al. "El Surgimiento del Derecho Administrativo Global..." op. cit. 7.
} 
Se trata, en cualquier caso, de una definición por enumeración que no agota todas las posibilidades (no es exhaustiva), y tampoco permite identificar con certeza si un determinado órgano es o no parte de la Administración global.

Una enumeración similar es la que realiza Pablo FernándeZ LAMELA cuando se refiere a "las redes transgubernamentales que están jugando un rol cada vez más importante en la regulación internacional...estas redes constituyen alianzas informales entre agencias gubernamentales, organizaciones internacionales, empresas transnacionales y participantes de la sociedad civil, como organizaciones no gubernamentales, corporaciones profesionales o grupos religiosos que se reúnen para analizar o discutir diversos temas de interés común." 30

Todas ellas serían, de conformidad con lo dicho hasta aquí, sujetos reguladores del Derecho Administrativo Global; esas administraciones globales "conforme a los poderes que tienen conferidas en el marco regulatorio de su actividad, que conforma las fuentes del derecho administrativo global, pueden adoptar decisiones que tienen efectos jurídicos directos respecto de los individuos, a veces, sin intervención alguna de las administraciones públicas nacionales e, incluso, en contra de ellas, configurándose aquellos, entonces, como sujetos de la Administración global." ${ }^{31}$

B. Sujetos de regulación. Una vez identificados los sujetos cuyas regulaciones contribuyen a configurar el Derecho Internacional Administrativo, corresponde identificar su contraparte, es decir, los órganos, instituciones o incluso personas sobre las cuales recae la obligación de cumplir aquellas regulaciones; en

\footnotetext{
30 Cfr. Fernández Lamela, P. “Globalización y Derecho público.... "op. cit. p. 53.

31 El campo de las administraciones globales progresivamente se ha ido desplazando hacia otros campos de actividad más vinculadas con aspectos sustantivos del tradicional derecho administrativo, como la protección ambiental, la seguridad internacional, la energía nuclear, el control de armas químicas, la propiedad intelectual y el régimen de las personas como por ejemplo el tratamiento de los refugiados. Cfr. Brewer-Carías, Allan R. "El Derecho administrativo global..." op. cit. pp. 4 y 5.
} 
el entendido de que para que las diferentes formas de administración global sean efectivas y sus regulaciones sean eficaces, requieren en primer término la existencia de unos sujetos que debieran acatarlas.

En las relaciones tradicionales entre el Derecho Administrativo y el Derecho Internacional esos sujetos están previamente determinados, puesto que al incorporar el contenido normativo del tratado al derecho interno aquél se hace directamente aplicable por las autoridades u órganos estatales correspondientes; de esa manera, no hay una ruptura en el encadenamiento entre las regulaciones administrativas internacionales y las internas.

La pertinencia analítica de los estudios de Derecho Administrativo Global con respecto a los sujetos de regulación radica en que toma como objeto de estudio los casos cada vez más frecuentes, de acatamiento y aplicación de regulaciones administrativas de origen internacional que no derivan necesariamente de las fuentes convencionales como los tratados internacionales.

La distinción sobre la cualidad de los sujetos de regulación se puede establecer mejor por el método de contraste: en las relaciones entre el Derecho Administrativo Internacional y el interno, los entes sometidos a su jurisdicción tienen carácter nacional, y sólo son relevantes al momento de la implementación de las regulaciones administrativas internacionales; por el contrario, en el Derecho Administrativo Global los destinatarios reales de las regulaciones son "cada vez más los mismos que en el derecho doméstico: a saber, los individuos (tanto como agentes morales y como actores económicos y sociales) y las entidades colectivas como las sociedades comerciales y, en algunos casos, las ONGs." 32

El punto está en que fuera del Derecho Administrativo Global los particulares o los agentes privados solo son relevantes para el Derecho Administrativo interno; mientras que en el Derecho Administrativo Global tanto los Estados como las

32 Kingsbury, B. et al. "El Surgimiento del Derecho Administrativo Global..." op. cit. p. 12. 
instituciones públicas o privadas y los ciudadanos se convierten en sujetos de derecho internacional; estos serían "los sujetos pasivos del derecho administrativo global, que en este caso no sólo son los Estados nacionales sino los individuos o ciudadanos de los mismos (administrados)." 33

Ahora bien, ¿Cuáles son, concretamente, esos sujetos de regulación del Derecho Administrativo Global, aquellos que se ven obligados a cumplir las disposiciones de los sujetos reguladores? Como en el caso de los primeros, tampoco los estudios de Derecho Administrativo Global disponen de una respuesta general a esta interrogante, puesto que además de las autoridades de administración formales del Derecho Administrativo interno, los autores sugieren casos de cumplimiento de aquellas regulaciones por parte de empresas privadas y aún de individuos, sin que haya necesariamente una mediación estatal.

Por lo dicho, se puede concluir que en los estudios sobre el Derecho Administrativo Global, se describe la expansión de los sujetos tradicionales del Derecho Administrativo en sus relaciones con el Derecho Internacional, al integrarse como sujetos de tales relaciones, además del Estado y las instituciones públicas de carácter administrativo, entidades privadas e incluso los ciudadanos, tradicionalmente excluidos en los estudios acerca de las relaciones entre Derecho Administrativo interno y el Derecho Internacional.

A falta de una delimitación clara de los sujetos del Derecho Administrativo Global, una manera más adecuada de abordar el tema es analizando los efectos de aquellas regulaciones en el Derecho Administrativo interno, así como la manera en que son incorporadas a las prácticas y procedimientos del Derecho Administrativo nacional; así, la pertinencia práctica del Derecho Administrativo Global depende de que las regulaciones de los entes identificados como parte de la Administración Global sean acatadas y aplicadas por los sujetos a quienes se dirigen, o aún

33 Cfr. Brewer-Carías, A. R. "El Derecho administrativo global... " op. cit. p. 5. 
por aquellos que sin estar obligados a su cumplimiento, prefieren hacerlo por los beneficios probables de su implementación.

\section{I.3. Formas de la regulación administrativa global}

Una vez analizados el sujeto regulador y los sujetos de regulación en el Derecho Administrativo Global, es preciso identificar las diferentes formas en que las regulaciones jurídicas de aquellos entes se manifiestan, lo que permitirá tener criterios mínimos para identificar, ante una norma concreta de Derecho Internacional, si puede ser considerada como perteneciente al Derecho Administrativo Global, o a las formas tradicionales de relación entre el Derecho Internacional y el interno.

En la base de ese análisis se encuentra la distinción que es ya un lugar común, entre hard law y soft law, expresiones con las que se hace referencia a dos formas distintas de manifestarse el Derecho Internacional; existen varios criterios y puntos de vista para diferenciar uno y otro tipo de derecho, ${ }^{34}$ aunque el punto de ruptura para la mayoría es su carácter vinculante o no para los sujetos a quienes se dirigen las regulaciones.

Al hard law se le define como el derecho con efectos vinculantes u obligatorios, mientras que el soft law significa derecho blando o derecho débil, entendido como no vinculante o no obligatorio. En su sentido jurídico, ambos conceptos designan el alcance que tiene el Derecho Internacional al definir y reglamentar las relaciones entre los Estados. El primero se refiere esencialmente a los tratados, convenios o convenciones internacionales que, una vez firmados y ratificado por los Estados siguiendo los procedimientos previstos en el derecho interno, se convierten en ley y, por tanto, adquieren fuerza vinculante.

Por el contrario, el soft law se define por exclusión con respecto a las fuentes en que se expresa el hard law; es decir,

\footnotetext{
34 Un análisis sistemático al respecto puede verse en Shaffer, Gregory C. y Pollack, Mark A. "Hard vs. Soft Law: Alternatives, Complements, and Antagonists in International Governance." Minnesota Law Review Vol 94/2010, pp. 706- 799.
} 
no se expresa a través de tratados, convenios o convenciones firmados o ratificados por el Estado, sino a través de declaraciones, resoluciones, acuerdos o programas de acción, que si bien deben estar en conformidad con el Derecho Internacional, no crean obligaciones legales o vinculantes para el Estado; su contenido es de índole indicativo y persuasivo, pueden tener el carácter de reglas técnicas o de estándares de optimización, cuyo uso o aplicación tiene efectos legales.

No obstante, las distinciones que se puedan realizar, los límites entre uno y otro tipo de derecho se han ido desdibujando con el tiempo, en lo que han influido tanto la ampliación permanente de los sujetos reguladores a nivel global como las formas concretas de instrumentación de las regulaciones internacionales por la administración interna o por la jurisprudencia.

Por otra parte, las potencialidades de la distinción también se utilizan en las relaciones internacionales por los Estados para posicionarse en la comunidad internacional en función de sus intereses, cuando no puede conseguir consenso para regulaciones vinculantes, se apela a las virtudes del soft law para llegar a acuerdos no vinculantes ab initio, pero que luego al ser aplicados por los demás Estados se convierten paulatinamente en vinculantes.

Luego de las distinciones de rigor, los estudios hacen mayor énfasis en analizar el soft law como categoría más problemática puesto que, como se ha dicho, debe ser definido bien por contraposición con el hard law, o bien a partir de la instrumentación administrativa o aplicación judicial de regulaciones no derivadas de instrumentos de Derecho Internacional vinculantes para los Estados.

Al margen de los desacuerdos teóricos sobre su definición, existen algunos puntos de convergencia entre diferentes autores, respecto a los criterios que se deben tener en cuenta para identificar si una regulación concreta se corresponde o no con el soft law; entre esos criterios se pueden mencionar los siguientes: 
i) que sean formulados en términos no obligatorios de acuerdo con los procesos tradicionales de creación de derechos;

ii) que contengan términos vagos e imprecisos;

iii) que procedan de órganos carentes de autoridad para dictar normas internacionales;

iv) que estén dirigidos a actores no estatales;

v) que sean ajenos a cualquier teoría de la responsabilidad;

vi) que estén basados exclusivamente en la adhesión voluntaria y no existan mecanismos para su exigibilidad. ${ }^{35}$

La estrategia adoptada por los autores pone de manifiesto las dificultades teóricas de hacer una definición exhaustiva, sistemática y coherente del soft law, aunque sí es posible identificar rasgos comunes a las concepciones más elaboradas sobre el tema; algunos de los puntos convergentes en cuanto a sus características son resumidos por Gloria Alarcón García en su estudio "El soft law y nuestro sistema de fuentes." ${ }^{36}$

Según la autora algunos puntos de coincidencia son: que el fenómeno del soft law aparece cuando el instrumento de regulación internacional tiene carácter jurídicamente no vinculante y posee cierta relevancia jurídica; se aplica respecto de instrumentos cuya juridicidad es dudosa o cuya fuerza vinculante se cuestiona; y se caracteriza por la no aplicabilidad de sanciones como consecuencia de su incumplimiento (no es sancionable mediante las formas tradicionales, pero existe lo que se viene denominando la soft coerción, la cual tiene sus propios canales de sanción).

Además de sus características estructurales, el soft law cumple funciones específicas con respecto a su contraparte el hard

35 Cfr. Chinkin, C. "Normative Development in the international legal system." Shelton, D. (ed), Commitment and compliance. The role of non-binding norms in the international legal system, New York, Oxford University Press, 2000, pp. 30- 31.

36 Alarcón García, G. "El soft law y nuestro sistema de fuentes", en: Tratado sobre la Ley General Tributaria. Libro-Homenaje del profesor Alvaro Rodríguez Bereiso, Madrid, Thomson Reuters-Aranzadi, 2010, pp. 271- 299. 
law; su uso estratégico en las relaciones internacionales cuando no es posible alcanzar un acuerdo vinculante lo convierte en un paso de avance hacia futuras regulaciones obligatorias entre los Estados; también funciona como una alternativa al hard law cuando no es posible conseguir acuerdos obligatorios, con respecto al cual puede funcionar como complemento o, en último lugar, constituir un parámetro interpretativo del hard law a los fines de su aplicación en sede administrativa o judicial. ${ }^{37}$

Por sus características, el soft law debe ser estudiado como fuente del Derecho Internacional a partir de su aplicación administrativa o judicial en el ordenamiento jurídico interno, y por ello el principal criterio para determinar su incidencia no es el de su origen, sino el de su vigencia efectiva en diferentes ramas del Derecho. Una de esas ramas en las que tiene influencia significativa el soft law es el Derecho Administrativo, ${ }^{38}$ por cuya influencia se ha ido desarrollando el Derecho Administrativo Global objeto de análisis en esta investigación.

De conformidad con lo explicado hasta aquí, se puede afirmar que las formas concretas de expresión del Derecho Administrativo Global están constituidas principalmente por las regulaciones propias del soft law, ${ }^{39}$ entre las que se encuentran las resoluciones, recomendaciones, declaraciones o textos programáticos de las instituciones y organismos internacionales respecto de las cuales no existe un tratado o convenio vinculante.

En segundo lugar, aparecen las recomendaciones, resoluciones, manuales de buenas prácticas y regulaciones de actores no estatales o público-privados que establecen estándares no

37 Cfr. Alonso García, R. "El soft law comunitario." Revista de Administración Pública, No. 54/2001, Madrid, pp. 74 y siguientes.

38 Cfr. la obra colectiva El soft law administrativo. Un estudio de los efectos de las normas no vinculantes de la administración. Prólogo de José Esteve Pardo. Thomson Civitas, Madrid, 2008.

39 Un estudio sobre las principales formas de expresión del soft law puede verse en MAZUELOS Bellido, Ángeles. "Soft Law, ¿Mucho ruido y pocas nueces?" Revista Electrónica de Estudios Internacionales, No. 8/ 2004, pp.1-40. 
obligatorios para la Administración pública interna, los entes privados y en algunos casos para los ciudadanos; en este caso el soft law sería "la única alternativa para los actores no estatales, dado que no tienen capacidad para crear normas y obligaciones jurídicas internacionales." ${ }^{40}$

También son forma de expresión del Derecho Administrativo Global los acuerdos internacionales no normativos y el llamado soft law material; esos "acuerdos son vinculantes, si bien no jurídicamente, dependiendo tal circunstancia de la voluntad de las partes. Como la voluntad de las partes de concluir un acuerdo jurídico o extrajurídico viene sólo excepcionalmente expresada, es necesario deducirla de una serie de índices de evaluación conjunta, entre los cuales podemos mencionar junto con el lenguaje, la denominación del texto, las partes que lo concluyen, la presentación del texto, las formalidades seguidas para su adopción, las cláusulas finales, la previsión o no de mecanismos de arreglo pacífico de controversias o la práctica subsiguiente." 41

Cualquiera que sea el origen de las regulaciones internacionales propias del soft law, o el contenido concreto que regulen, lo importante es que se trata de resoluciones, documentos o guías programáticas cuyo contenido no es de obligatorio cumplimiento para sus destinatarios, sean los Estados, organizaciones o empresas privadas o los ciudadanos; de ahí que se pueda afirmar que "el foco del derecho administrativo global no es el contenido específico de las reglas sustantivas, sino el funcionamiento de principios existentes o posibles, de reglas procedimentales, de mecanismos de revisión y de otros mecanismos relativos a la transparencia, participación, toma de decisiones razonadas y a garantizar la legalidad en la gobernanza global.”42

\footnotetext{
40 Mazuelos Bellido, Á. "Soft Law...?” op. cit. p. 13.

41 Mazuelos Bellido, Á. "Soft Law...?" op. cit. p. 16- 17.

42 Cfr. Kingsbury, B. et al. op. cit. p. 17.
} 


\section{I.4. Las normas de Derecho Administrativo Global como fuente de derecho interno}

La afirmación anterior remite al estudio de las fuentes del Derecho Administrativo interno y, más concretamente, al de las relaciones entre el Derecho Administrativo Global y su recepción por los sujetos públicos y privados, o por los ciudadanos, como fuente para la aplicación o resolución de conflictos administrativos internos.

La relación más evidente que se puede establecer entre el Derecho Internacional y el Derecho Administrativo nacional es que las disposiciones jurídicas de aquél deben ser acatadas por las autoridades nacionales, a través de los mecanismos legalmente existentes para la recepción y aplicación de los tratados internacionales.

Sin embargo, como se ha explicado, el Derecho Administrativo Global va un paso más allá de esas relaciones formales, puesto que, al ampliarse los órganos de regulación a sujetos no legitimados mediante un tratado, tales mecanismos se tornan insuficientes.

Es por ello por lo que, en este punto, una de las cualidades más importantes del Derecho Administrativo Global es la existencia de sujetos regulatorios cuyas disposiciones son acatadas y cumplidas por autoridades nacionales, independientemente de la existencia o no de un tratado vinculante al respecto, y es ahí donde entra el tema de las fuentes del Derecho Administrativo, uno de los tópicos más recurrentes en los textos sobre la materia.

En dichos textos, los análisis relativos a las fuentes del derecho no hacen referencia a lo que hasta aquí se ha identificado como Derecho Administrativo Global, aunque sí a los tratados internacionales en la mayoría de los casos. Así Miguel MARIENHOFF considera que por fuentes del derecho administrativo, deben entenderse los diversos orígenes posibles de las normas y principios que interesan al derecho administrativo, tengan o 
no eficacia normativa expresamente establecida (incluye acá la Constitución, leyes dictadas en consecuencia de la Constitución, los tratados con potencias extranjeras y los reglamentos administrativos; también son fuentes la analogía, los principios generales del derecho, la jurisprudencia y la doctrina científica). ${ }^{43}$

Para Cassagne, las fuentes del derecho son los medios o formas que dan origen al ordenamiento jurídico, distinguiéndose entre las fuentes en reales (o materiales) y formales: las primeras son aquellos modos (elementos, factores, etc.) que determinan el contenido de las normas jurídicas, con independencia de los órganos con competencia para producir disposiciones jurídicas; mientras que las segundas son aquellas que dan origen al ordenamiento, y que emanan de órganos estatales competentes, en este sentido el concepto fuente se limita a la constitución, la ley y el reglamento. ${ }^{44}$

Fernando Garrido Falla entiende por fuentes del Derecho Administrativo "aquellas formas o actos a través de los cuales el Derecho Administrativo se manifiesta en su vigencia; (fuentes escritas: la Constitución, la ley, los tratados internacionales, los reglamentos estatales, y los reglamentos dictados por otras corporaciones públicas sometida a tutela estatal; fuentes no escritas: la costumbre y los principios generales del derecho; y fuentes indirectas: los tratados internacionales y la jurisprudencia) ${ }^{45}$ y según Martín Mateo, las fuentes "no son otra cosa que el procedimiento de originación de normas jurídico-administrativas clasificándolas en fuentes primarias, las que nos dan un derecho directamente aplicable per se (leyes, reglamentos y tratados internacionales); fuentes complementarias, cuya vigencia se deriva de los propios pronunciamientos de las fuentes primarias (costumbre y principios generales del

\footnotetext{
43 Marienhoff, M. S. op. cit. p. 105- 107.

44 Cassagne, J. C. Derecho Administrativo. Editorial LexisNexis/Abeledo- Perrot, Buenos Aires, 1998, p. 123- 125.

45 Garkido Falla, F. Tratado de Derecho Administrativo Volumen I, parte General. Duodécima edición, Editorial Tecnos, 1994, pp. 171- 174.
} 
derecho) y fuentes aclaratorias, orientan sobre el auténtico sentido y alcance de lo querido por el legislador (jurisprudencia y doctrina científica)." ${ }^{46}$

Del recuento anterior, interesa destacar por un lado la variedad de definiciones posibles y, por otro, los dos criterios asumidos por los autores para conceptualizar las fuentes del Derecho Administrativo: el origen de las normas administrativas, al concebir literalmente el término "fuente", y su vigencia, entendida como manifestación de su aplicación efectiva. El Derecho Administrativo tradicional, circunscrito a la soberanía estatal, es insuficiente para dar cuenta de la influencia que las regulaciones de las autoridades globales, públicas o privadas tienen en la Administración pública interna; esa insuficiencia es notable cuando se identifican las fuentes del Derecho Administrativo, entre las que no aparecen las regulaciones propias del Derecho Administrativo Global.

Como puede apreciarse, se trata de obras publicadas en las últimas décadas, mismas en que se ha ido desarrollando el Derecho Administrativo Global y que, al parecer, no han tenido ninguna influencia en la configuración de las fuentes tradicionales del Derecho Administrativo; lo más parecido a una influencia de ese tipo es la consideración de los tratados internacionales como fuentes, sin embargo deben pasar, para su efectiva vigencia, por el proceso de recepción establecido en el ordenamiento jurídico.

Para acreditar la veracidad de la afirmación anterior deben identificarse, bien sea en general o mediante ejemplos concretos, las que pueden considerarse fuentes del Derecho Administrativo Global, para analizar si pueden ser consideradas o son de hecho fuentes del Derecho Administrativo interno, tomando en cuenta el criterio de vigencia efectiva y no el de su origen o forma de integración al ordenamiento jurídico nacional.

46 Martín Mateo, R. Manual de Derecho Administrativo. 22 ed., Aranzadi, Pamplona, 2003, pp. 77- 84 . 
CASSESE considera que existe un ordenamiento jurídico global basado en la cooperación y que carece de soberanía, pues funciona con base en un tipo de organización diferente al Estado tradicional; en ese contexto hay una comunidad o sociedad internacional en la que están presentes los tres elementos del ordenamiento jurídico: plurisubjetividad (principalmente los Estados), organización (principalmente organizaciones internacionales) y normativa (la mayor parte, convencional o pactada, aunque también reglamentaria). Ese ordenamiento jurídico internacional "no se rige por una autoridad superior, sino que se basa en la cooperación y en la forma de integrarse los sujetos que forman parte de él..." ${ }^{77}$ "a esa característica se añade ahora que su organización no tiene un centro de referencia. Es, sin embargo, una agrupación de organizaciones generales y sectoriales." 48

Las fuentes, en sentido formal, que integran ese ordenamiento jurídico global, son "los principios constitucionales o fundamentales, que proceden de las tradiciones nacionales, las costumbres y los tratados, a las que se añaden aquellas denominadas de varias formas, que están producidas por las organizaciones internacionales, dirigidas a los Estados o a comunidades subestatales, las reconocidas globalmente, pero producidas por comunidades sectoriales individuales, como las que conciernen al Derecho comercial y de los contratos, las de protección de los consumidores, seguridad, condiciones de trabajo, responsabilidad, tutela del medio ambiente y las de transparencia y participación." 49

Ese sistema de normas presenta- según CASSESE- cuatro características fundamentales: está constituido por un gran número de preceptos indicativos, no imperativos; produce una

\footnotetext{
47 CASSESE, S. "El espacio jurídico global." Revista de Administración Pública No. 157/ 2002 p. 15, pp. 11- 26.

48 Cassese, S. "El espacio jurídico..." op. cit. p. 16.

49 Cassese, S. "El espacio jurídico..." op. cit. pp. 20- 21.
} 
gran variedad de efectos laterales, porque carece de un límite territorial; se presta, por la ausencia de una división rígida de las tareas, a usos encaminados a alcanzar efectos colaterales; está compuesto por normas de diferente procedencia, supranacional y nacional, por lo que puede decirse que el derecho global no coincide con el Derecho Internacional: este último es sólo una parte del primero; finalmente el ordenamiento jurídico global está dotado de disposiciones e instrumentos para las soluciones de controversia." 50

Ante la imposibilidad de una definición de las fuentes del Derecho Administrativo Global por su origen o su forma, el criterio más plausible para considerarla dentro de las fuentes del derecho interno sería el de su vigencia y eficacia en el ordenamiento jurídico nacional. Se trata en todo caso de un criterio empírico que permitiría determinar si una regulación concreta de uno de los sujetos reguladores del Derecho Administrativo Global es acatada y cumplida por las autoridades internas, sobre todo por aquellas con respecto a las cuales la entidad reguladora global no está vinculada a través de un tratado o convenio internacional.

Con ello se reduciría por un lado el carácter principalmente teórico de los estudios actuales sobre el Derecho Administrativo Global, al tiempo que haría posible la verificación de si, más allá de las afirmaciones teóricas sobre su influencia en el derecho interno, en un ordenamiento jurídico determinado las decisiones de los entes reguladores globales son aplicadas por los entes internos, aunque no haya una norma vinculante al respecto.

En este sentido es necesario tomar en consideración, como sostiene Darnaculleta Gardella, que, en un número creciente de sectores, los organismos transnacionales de regulación ejercen el monopolio de la producción de estándares internacionales, en una suerte de delegación de la autoridad regulatoria en el ámbito global, que son aplicados por los Estados con independencia

50 Cassese, S. "El espacio jurídico..." op. cit. pp. 21- 22. 
de su validez jurídica, o son simplemente incorporados como derecho en los ordenamientos jurídicos nacionales. ${ }^{51}$

Son numerosos los organismos normativos mundiales cuyos estándares penetran directamente en el contexto regulador nacional y son asumidos y aplicados por entidades nacionales, respetadas en la práctica pese a no ser formalmente vinculantes; precisamente por las presiones del entorno altamente competitivo y por la supervisión constante de su cumplimiento. ${ }^{52}$

La posición más relevante la ocupa la regulación del sector financiero, ${ }^{53}$ en el que destacan las llamadas agencias de rating que, concebidas inicialmente para solventar las dificultades del acceso a la información financiera en el mercado, han alcanzado un enorme poder en el escenario financiero global, al punto de ser capaces de alterar el equilibrio financiero y monetario internacional y condicionar las agendas políticas de los Estados y las organizaciones internacionales, pudiendo incluso provocar consecuencias desastrosas a escala global. ${ }^{54}$

Por otro lado, de manera creciente son los propios actores privados los destinatarios de la acción administrativa de los organismos globales a través de la adopción de medidas o realización de acciones ejecutivas con efectos jurídicos inmediatos, sin necesidad en algunos casos de intervención de autoridades nacionales, -p. ej., el Consejo de Seguridad de la ONU y sus comités, que adoptan decisiones en materia de seguridad internacional, tanto respecto de países concretos (fundamentalmente sanciones) como sobre las personas (la lista de personas consideradas amenazas a la paz mundial, la determinación de

\footnotetext{
51 Darnaculleta Gardella, M. M. “El Derecho Administrativo Global. ¿Un nuevo concepto clave del Derecho administrativo?" Revista de Administración Pública, No. 199/2016, p. 24.

52 Marcheco Acuña, B. “Los desafíos..., ob. cit., pp. 7-8.

53 Un análisis sobre la regulación global en el sector financiero puede verse en: DARNACULLETA Gardella, María M. y Salvador Armendáriz, María A. "Nuevas fórmulas de génesis y ejecución normativa en la globalización: el caso de la regulación de la actividad financiera», Revista de Administración Pública, No. 183, 2010, pp. 139-177.

54 SIRIANNI, G. «ll rating sovrano», Constitucionalismo.it, Fascicolo 2, 2012, p.1.
} 
la condición de refugiado, etc.); o las sanciones impuestas a atletas, clubes o federaciones nacionales por organismos deportivos mundiales; el reconocimiento internacional de una marca; etc.-, con lo cual además se va reduciendo progresivamente la distancia que entre aquéllos y éstos había impuesto el Derecho internacional público tradicional.

Entre la actividad normativa o administrativa global y el derecho nacional se producen inevitables tensiones como consecuencia de la disparidad y heterogeneidad de las normas internacionales y los cuestionamientos sobre la legitimidad de origen de muchas de ellas, toda vez que éstas no son producto de un ordenamiento jurídico unitario con una estructura jerárquica de las fuentes que aseguren su unidad y coherencia, ni cuentan muchas de ellas en su producción o aplicación con las garantías (procedimentales, de control o tutela) propias de los ordenamientos nacionales.

Estas tensiones serán más intensas en la medida en que las normas o las acciones administrativas de instancias internacionales afecten directamente la esfera de los derechos, y, aunque difícilmente puedan ser resueltas del todo, si pudieran ser atenuadas a través de los métodos de interpretación del Derecho y la introducción de fórmulas correctivas.

\section{Conclusiones}

1. El sentido y alcance del Derecho Administrativo Global (identificado también en la doctrina como Derecho Administrativo Internacional), como una reciente proyección del Derecho Administrativo (concebido tradicionalmente como derecho público interno) afectado por el fenómeno de la globalización; abarca las normas y las instituciones internacionales cuyas regulaciones (sean tratados internacionales o no) irradian en la Administración pública interna.

2. En los estudios sobre el Derecho Administrativo Global, se describe la expansión de los sujetos tradicionales del Derecho 
Administrativo en sus relaciones con el Derecho Internacional, al integrarse como sujetos de tales relaciones, además del Estado y las instituciones públicas de carácter administrativo, entidades privadas e incluso los ciudadanos, tradicionalmente excluidos en los estudios acerca de las relaciones entre Derecho Administrativo interno y el Derecho Internacional.

3. El Derecho Administrativo Global se manifiesta, básicamente, a través de normas jurídicas de tipo soft law, entre las que se encuentran resoluciones, recomendaciones, manuales de buenas prácticas, declaraciones o textos programáticos de las instituciones y organismos internacionales respecto de las cuales no existe un tratado o convenio vinculante.

4. Las normas de Derecho Administrativo Global pueden ser fuente de Derecho Administrativo interno, en la medida en que una regulación concreta de uno de los sujetos reguladores del Derecho Administrativo Global sea acatada y cumplida por las autoridades internas, sobre todo por aquellas con respecto a las cuales la entidad reguladora global no está vinculada a través de un tratado o convenio internacional.

\section{Bibliografía}

Alonso García, Ricardo. "El soft law comunitario." Revista de Administración Pública, No. 54/2001, Madrid, pp. 63-94.

A Dictionary of Law. Oxford University Press, 5ta edición 2003.

AA.VV. El soft law administrativo. Un estudio de los efectos de las normas no vinculantes de la administración. Prólogo de José Esteve Pardo. Thomson Civitas, Madrid, 2008.

Alarcón García, Gloria. "El soft law y nuestro sistema de fuentes." En Tratado sobre la Ley General Tributaria. Libro-Homenaje del profesor Álvaro Rodríguez Bereijo, Thomson ReutersAranzadi 2010, pp. 271- 299.

BARNÉs, Javier. "Transformaciones (científicas) del Derecho administrativo. Historia y retos del Derecho administrativo contemporáneo." Disponible en http://es.globallawpress.org/ wp-content/uploads/Transformaciones-cient\%C3\%ADficas- 
del-Derecho-Administrativo.-Historia-y-retos-del-DerechoAdministrativo-contempor\%C3\%A1neo.pdf. Consultado el 6 de julio de 2018.

BAssiouni, Cherif. "El Derecho penal internacional: Historia, objeto y contenido." Anuario de Derecho Penal y Ciencias Penales, Madrid, 1982, pp. 5- 42.

Brewer-Carías, Allan R. "El Derecho administrativo global en materia de cooperación policial y el procedimiento administrativo global desarrollado ante interpol, como administración global, para la protección de las personas." Ponencia en el Tercer Congreso Internacional de Derecho Administrativo, sobre "Derecho Administrativo Global" organizado por el Instituto de Investigaciones Jurídicas de la Universidad Nacional Autónoma de México y la Facultad de Derecho y Criminología de la Universidad Autónoma de Nuevo León, Monterrey, Nuevo león, 23-25 abril 2009, p 3 y siguientes. Disponible en http:// allanbrewercarias.com/wp-content/uploads/2009/04/1045.1-990.-PROCEDIMIENTO-ADMINISTRATIVO-GLOBALEN-MATERIA-DE-POLIC\%C3\%8DA.-Monterrey.doc.pdf. Consultado el 5 de julio de 2018.

Cassagne, Juan Carlos. Derecho Administrativo. Editorial LexisNexis/ Abeledo- Perrot, Buenos Aires, 1998.

Cassese, Sabino. "El espacio jurídico global." Revista de Administración Pública No. 157/ 2002, pp. 11- 26.

Cassese, Sabino. The global polity. Global dimensions of democracy and the rule of law, Sevilla, Global Law Press, 2012, p. 175.

Cassesse, Sabino. "New paths for administrative law: A manifesto", International Journal of Constitutional Law, Vol. 10(3)/2012, p. 605.

Chávez, María del Pilar. "La inexistencia del derecho administrativo global." Diario Administrativo No. 76 de 4 de agosto de 2015. Disponible en https://dpicuantico.com/sitio/wp-content/ uploads/2015/08/Administrativo-Doctrina-2015-08-04.pdf. Consultado el 6 de julio de 2018.

Chinkin, C. "Normative Development in the international legal system." Shelton, D. (ed), Commitment and compliance. The role of non-binding norms in the international legal system, New York, Oxford University Press, 2000, pp. 30- 31. 
Darnaculleta Gardella, María M. "El Derecho Administrativo Global. ¿Un nuevo concepto clave del Derecho administrativo?" Revista de Administración Pública, No. 199/2016, p. 24.

Decreto- Ley No. 317/2013, "De la Prevención y Detección de Operaciones en el Enfrentamiento al Lavado de Activos, al Financiamiento al Terrorismo, a la Proliferación de Armas y al Movimiento de Capitales Ilícitos." GOC-2018-182-EX25, de 26 de abril de 2018.

Escuin Palop, Catalina. Curso de derecho administrativo parte general. Tirant lo Blanch, Valencia, 2011.

Esteve Pardo, José. Prólogo a El soft law administrativo. Un estudio de los efectos de las normas no vinculantes de la administración. Thomson Civitas, Madrid, 2008.

EstupiÑan SILVA, Rosmerlin. "Principios que rigen la responsabilidad internacional penal por crímenes internacionales." UNAMInstituto de Investigaciones Jurídicas. Anuario Mexicano de Derecho Internacional, vol. XII, 2012, pp. 133-173.

Fernández Lamela, Pablo. "Globalización y Derecho público. Introducción al Derecho administrativo internacional.” En CiENFuEgos Salgado, David y López Olvera, Miguel Alejandro (coords.) Estudios en homenaje a Don Jorge Fernández Ruiz. Tomo I Derecho Administrativo, UNAM, 2005, pp. 45- 63.

Fernández RozAs, José Carlos. "El Derecho del comercio internacional en el contorno de la globalización.” Escriva. Revista del Colegio de Notarios del Estado de México, No. 5/2000, pp. 161-230.

Gambardella d'Etigny, Maite y von Loebenstein Weil, Beatriz. Recensión al libro Hacia el Derecho Administrativo Global: fundamentos, principios y ámbito de aplicación de Benedict KingsBury y Richard B. Stewart (Global Law Press, Sevilla, 2016). Revista de Derecho Público, Universidad de Chile, vol. 84/2016, p. 179. Pp. 1791- 183.

García de Enterría, Eduardo y Fernández, Tomás-Ramón. Curso de Derecho Administrativo I. Decimoquinta edición. Editorial Civitas/Thomson Reuters, 2011.

Garcini Guerra, Héctor. Derecho Administrativo. Editorial Pueblo y Educación, La Habana, 1986.

Garrido Falla, Fernando. Tratado de Derecho Administrativo Vol. I, parte General. Duodécima edición, Editorial Tecnos, 1994. 
Gordillo, Agustín. Los valores en el Derecho administrativo global. Disponible en http://www.derecho.uba.ar/docentes/pdf/ el-control-de-la-actividad-estatal/cae-gordillo-valores.pdf. Consultado el 6 de junio de 2018.

KIngsbury, Benedict, et al. "El Surgimiento del Derecho Administrativo Global." Revista de Derecho Público No. 24/2010, Facultad de Derecho, Universidad de los Andes, Colombia, pp. 4- 46.

López Escarcena, Sebastián. "Contextualizando el derecho administrativo global." Anuario Colombiano de Derecho Internacional, Vol. 11/2018, pp. 259-305.

López Escarcena, Sebastián. "Contextualizando el derecho administrativo..." op. cit. p. 261. Anuario Colombiano de Derecho Internacional, Vol. 11/2018, pp. 259-305.

Marcheco Acuña, Benjamin. "Los desafíos de la ciencia del Derecho administrativo ante la globalización", Revista General de Derecho Administrativo, No. 49, 2018.

Marienhoff, Miguel S. Tratado de Derecho Administrativo. Editorial Abelerdo- Perrot, Buenos Aires, 1983.

Martín Mateo, Ramón. Manual de Derecho Administrativo. 22' Edición, Editorial Aranzadi, 2003.

Matilla Correa, Andry. "Las fuentes del Derecho Administrativo (excepto el reglamento)". Temas De Derecho Administrativo Cubano, Editorial Félix Varela, 2004.

Muci Borjas, José Antonio. "Control Judicial y Arbitraje Internacional conforme al Derecho Administrativo Global.” Centro de Arbitraje de la Cámara de Comercio de Caracas, 2005, p. 11. Disponible en www.muci-abraham.com/.../c4054864837660d0350a7407afbbbc141b9832a9.pdf. Consultado el 6 de julio de 2018.

Richards Martínez, Orisel. "Una mirada a los presupuestos teóricos para la recepción de tratados internacionales en el ordenamiento jurídico cubano." Revista de la Facultad de Derecho y Ciencias Politicas de la Pontificia Universidad Bolivariana de Medellin, No. 116/2012, pp. 117- 158.

Rodríguez-Arana MuÑoz, Jaime. "El Derecho Administrativo Global: un derecho principal." Revista Andaluza de Administración Pública No. 70/2010, pp. 15- 68.

Ruzz, Julián Modesto. Principios de Derecho Administrativo. Escuela de Ciencias Comerciales, Universidad de la Habana, 1950. 
SARMiento, Daniel. "La autoridad del Derecho y la naturaleza del soft law." Cuadernos de Derecho Público, No. 22/2006, pp. 220- 266. Schmidt-Assmann, Eberhard. "La ciencia del derecho administrativo ante el reto de la internacionalización de las relaciones administrativas." Revista de Administración Pública No. 171/2006, Madrid, pp. 7-34.

Sentencia Grimaldi, de 13-12-1989, del Tribunal de Justicia de la Unión Europea.

SHAffer, Gregory C. y Pollack, Mark A. "Hard vs. Soft Law: Alternatives, Complements, and Antagonists in International Governance." Minnesota Law Review Vol 94/2010, pp. 706- 799.

STEWART, Richard. "The global regulatory challenge to U.S. administrative law". New York University Journal of International Law and Politics, Vol. 37/2005, p. 703.

Thürer, D. "Soft Law", en Bernhardt, R. (ed.), Encyclopedia of Public International Law, 2000. 